\title{
Accuracy of diffusion-weighted imaging in discriminating atypical vertebral haemangiomas from malignant masses in patients with vertebral lesions: a cross-sectional study
}

\author{
Parisa Hajalioghli ${ }^{1 A, B, F}$, Mohammad Hossein Daghighi ${ }^{1 A, B, E}$, Jaber Ghaffari ${ }^{1 B, C, D}$, \\ Mohammad Mirza-Aghazadeh-Attari ${ }^{1 B, C, E, F}$, Jhila Khamanian ${ }^{1 B, C, E, F}$, Payam Ghaderi1A,D,F, Iman Yazdaninia ${ }^{1 B, C, F}$, \\ Shadi Daghighi ${ }^{1,2 D, E, F}$, Armin Zarrintan ${ }^{1 C, D, E, F}$
}

'Department of Radiology, Faculty of Medicine, Tabriz University of Medical Sciences, Tabriz, Iran

¿University of California in San Diego, San Diego, California, USA

\section{Abstract}

Purpose: Vertebral haemangiomas are incidental findings in imaging modalities. Atypical haemangiomas are haemangiomas rich in vascular tissue, and they are found to be hypointense in T1 sequences and hyperintense in T2 sequences, mimicking the findings of metastatic lesions. In the present study we aim to evaluate the ability of diffusionweighted imaging to differentiate these two groups of vertebral lesions.

Material and methods: In the present cross-sectional study, a total of 23 lesions were included, including 10 haemangiomas and 13 malignant lesions. Diffusion-weighted imaging was used to compare atypical haemangiomas and metastatic lesions. The apparent diffusion co-efficient was determined for each lesion, and then the mean of each group was calculated. The means were then compared. Receiver operating characteristic analysis was used to determine a cut-off ADC value to differentiate these lesions.

Results: The difference between the mean age of the two groups was not significant. The mean ADC value for atypical haemangiomas was $1884 \pm 74 \times 10^{-6} \mathrm{~mm}^{2} / \mathrm{s}$ and $1008 \pm 81 \times 10^{-6} \mathrm{~mm}^{2} / \mathrm{s}$ for the malignant lesions. The difference between the two groups was statistically significant $\left(p<10^{-3}\right)$. ROC curve analysis determined an ADC value of $958 \times 10^{-6} \mathrm{~mm}^{2} / \mathrm{s}$ to be able to differentiate between atypical haemangiomas and malignant lesions.

Conclusions: Diffusion-weighted MRI could be used to differentiate between atypical haemangiomas and malignant metastatic lesions.

Key words: DW, haemangioma, metastasis, MRI, cancer.

\section{Introduction}

Haemangiomas of the vertebra are the most common benign tumours of the vertebra bodies; they are usually asymptomatic lesions and are unintentionally diagnosed amid imaging performed for other purposes [1]. Haemangiomas can be diagnosed by various imaging modalities, including plain radiographs and computed tomography (CT) scans. Axial CT scans can show the characteristic polka-dotted appearance, which is a series of thickened vertebral trabeculae [2]. Another imaging modality used is magnetic resonance imaging. Haemangiomas are shown as hyper-intense lesions in T1 and T2 sequences [3]. This is because of the high fat and water content of haemangiomas. These characteristic findings in magnetic resonance (MR) imaging and CT scan help differentiate haemangiomas from malignant lesions, including primary malignant lesions arising in the vertebra, infiltrative malignant lesions

Correspondence address:

Dr. Armin Zarrintan, Department of Radiology, Faculty of Medicine, Tabriz University of Medical Sciences, Tabriz, Iran, e-mail: zarrintanarmin@gmail.com

Authors' contribution:

A Study design · B Data collection · C Statistical analysis · D Data interpretation · E Manuscript preparation · F Literature search 
such as multiple myeloma, and more commonly distant metastasis [4,5]. Atypical haemangiomas are a group of haemangiomas with atypical histological characteristics. These lesions do not contain the high fat content of normal haemangiomas, and they have a rich vascular structure [6]. Although atypical haemangiomas are usually symptom free and less common than the typical kind, they are of clinical importance because they can be mistaken for malignant lesions in imaging modalities - this is because they are hypo intense in $\mathrm{T} 1$ sequences and hyper intense in $\mathrm{T} 2$, resembling metastasis [7]. Histopathologically, atypical haemangiomas do not resemble metastases, which comprise dense bodies of tightly packed small malignant cells. Atypical haemangiomas are made up of vascular spaces, with great intercellular distances. Scholars have suggested that diffusion-weighted imaging (DWI) may be beneficial in differentiating these lesions [8]. DWI relies on the ability of water molecules to have random movement in an acellular structure, thus enabling it to detect limited or increased diffusion of water molecules [9]. Previous studies have been able to show that malignant lesions do in fact have characteristic diffusion profiles and significantly altered apparent diffusion coefficient (ADC) values compared to normal tissue and benign lesions [10]. However, there is little evidence available regarding the efficacy of DW-MRI in differentiating haemangiomas, especially atypical vertebral haemangiomas, from distant metastasis of the vertebra. In this study, we aim to compare $\mathrm{ADC}$ values of atypical haemangiomas and metastasis of the vertebra in specific $b$ values, and to analyse whether there are significant differences between the two.

\section{Material and methods}

\section{Patients}

In the present cross-sectional study, subjects being referred to a tertiary medical educational centre between April 2016 and June 2019 were included. Subjects being included either had an atypical haemangioma or a metastasis in the vertebra, or any other malignant lesion in the vertebra. Inclusion criteria consisted of having an atypical haemangioma in the vertebra, diagnosed by documenting the polka-dot sign or corduroy sign in CT imaging; having a metastatic lesion in the vertebra, which was defined by either a biopsy, positive radio-nucleotide scan, or pet scan; having a hypointense or isointense lesion in T1 sequence; and being hyper intense in T2 sequences. Exclusion criteria consisted of patients having any sort of contraindication for MRI and lack of will to participate in the study.

\section{Magnetic resonance imaging}

All patients underwent imaging using a single machine (Avanto, 1.5 Tesla, Siemens-Erlangen, Germany). Imaging was done in supine position, with hands held still alongside the body. Imaging was performed in T1, T2, and DW sequences. Occasionally, other sequences were also investigated. T1 imaging was done according to the following specifications: sagittal plane, $\mathrm{FOV}=315 \mathrm{~mm}, \mathrm{TR}=762 \mathrm{~ms}$, $\mathrm{TE}=11 \mathrm{~ms}$, slice thickness $=4.5 \mathrm{~mm}$, and $\mathrm{T} 2$ imaging was performed according to the following specifications: $\mathrm{TR}=3723 \mathrm{~ms}, \mathrm{TE}=107 \mathrm{~ms}, \mathrm{FOV}=315 \mathrm{~mm}$, slice thickness $=4.5 \mathrm{~mm}$. DW imaging was performed in axial and sagittal planes, using the spin-echo single-shot echo-planar technique. Imaging was done in $\mathrm{b}$ values of 50 and 400 , using fat-suppression. Imaging was done using the following specifications: $\mathrm{TR}=25 \mathrm{~ms}, \mathrm{TE}=7 \mathrm{~ms}, \mathrm{FOV}=$ $400 \mathrm{~mm}$, slice thickness $=7 \mathrm{~mm}$.

\section{Image interpretation}

The imaging was performed by a single team of radiologists using a single MRI machine. A single team of expert radiologists were responsible for reporting imaging findings. The radiologists were not aware of the pathology reports of lesions, or any link to the final diagnosis.

Regions of interest (ROIs) for quantification of the witnessed values were defined manually using T1WI inand out-phase images, with and without fat suppression (once in T1) and DW images. A ROI in a non-pathologic vertebral bone marrow was also drawn manually, covering at least $50 \%$ of the area. Whole vertebral body was assessed in a similar manner. In pathologic lesions, All ROIs were selected from the central segments of lesions in the above sequence images (at least two thirds were covered). The size of the ROI changed based on lesion size, but most of the measurements were made by a region with a size of $0.5 \mathrm{~cm}^{2}$. To ensure the consistency, all measurements were performed three times with consistent size of ROI at different image levels, and average values were calculated.

\section{Statistical analysis}

Statistical analysis was done using SPSS version 24. Nonparametric tests, including Mann-Whitney test and twosample Kolmogorov-Smirnov test, were used for comparing the mean ADC values between atypical haemangiomas and metastasis. Unpaired two-tailed Student's $t$-test was also used in this regard. The sensitivities and specificities of the quantitative parameters for the detection of spinal metastases to be differentiated from haemangiomas was recorded and a receptor operative curve was drawn.

\section{Ethical considerations}

All patients included in the study signed a written informed consent form. This study was approved by the regional ethics board of the institution in which the study was performed. The study was in concordance with the latest edition of the Helsinki Declaration. 


\section{Results}

In total, 23 patients were enrolled in the study, with 10 of them having atypical vertebral haemangiomas and 13 having vertebral metastasis or other malignant lesions. In total, 17 patients were male and six were female. The mean age of patients was $53.39 \pm 3.17$ years with a minimum of 27 and maximum of 78 years. The mean age of patients with atypical haemangiomas was $51.18 \pm 4.16$ years, and it was $54.62 \pm 4.72$ years for the metastasis group. The difference between the two groups was not significant. From the 13 patients with malignant metastasis, seven had multiple myeloma, two had lung cancer, one patient had renal carcinoma, one had lymphoma, one had prostate cancer, and one had a metastasis from a germ cell tumour. Figures 1-3 show imaging findings of some of the patients included in the study.
The mean \pm standard deviation ADC value for patients with atypical haemangioma was $1884 \pm 74 \times 10^{-6} \mathrm{~mm}^{2} / \mathrm{s}$, and it was $1008 \pm 81 \times 10^{-6} \mathrm{~mm}^{2} / \mathrm{s}$ for the patients with malignant lesions. The difference between the two groups was statistically significant $\left(p<10^{-3}\right)$. The maximum and minimum ADC values recorded in the atypical haemangioma group were 2280 and $1600\left(\times 10^{-6} \mathrm{~mm}^{2} / \mathrm{s}\right)$, respectively, while they were 1850 and $680\left(\times 10^{-6} \mathrm{~mm}^{2} / \mathrm{s}\right)$ for the group with malignant lesions, respectively. The highest ADC value recorded in the malignant group belonged to a focal multiple myeloma. Figure 4 depicts the plots and whisker diagram of the two groups. Receiver operating characteristic analysis was done to determine an ADC value cut-off to differentiate atypical haemangiomas from metastasis. Figure 5 depicts the ROS curve generated. The cut-off value was set at $958 \times 10^{-6} \mathrm{~mm}^{2} / \mathrm{s}$. Figure 6
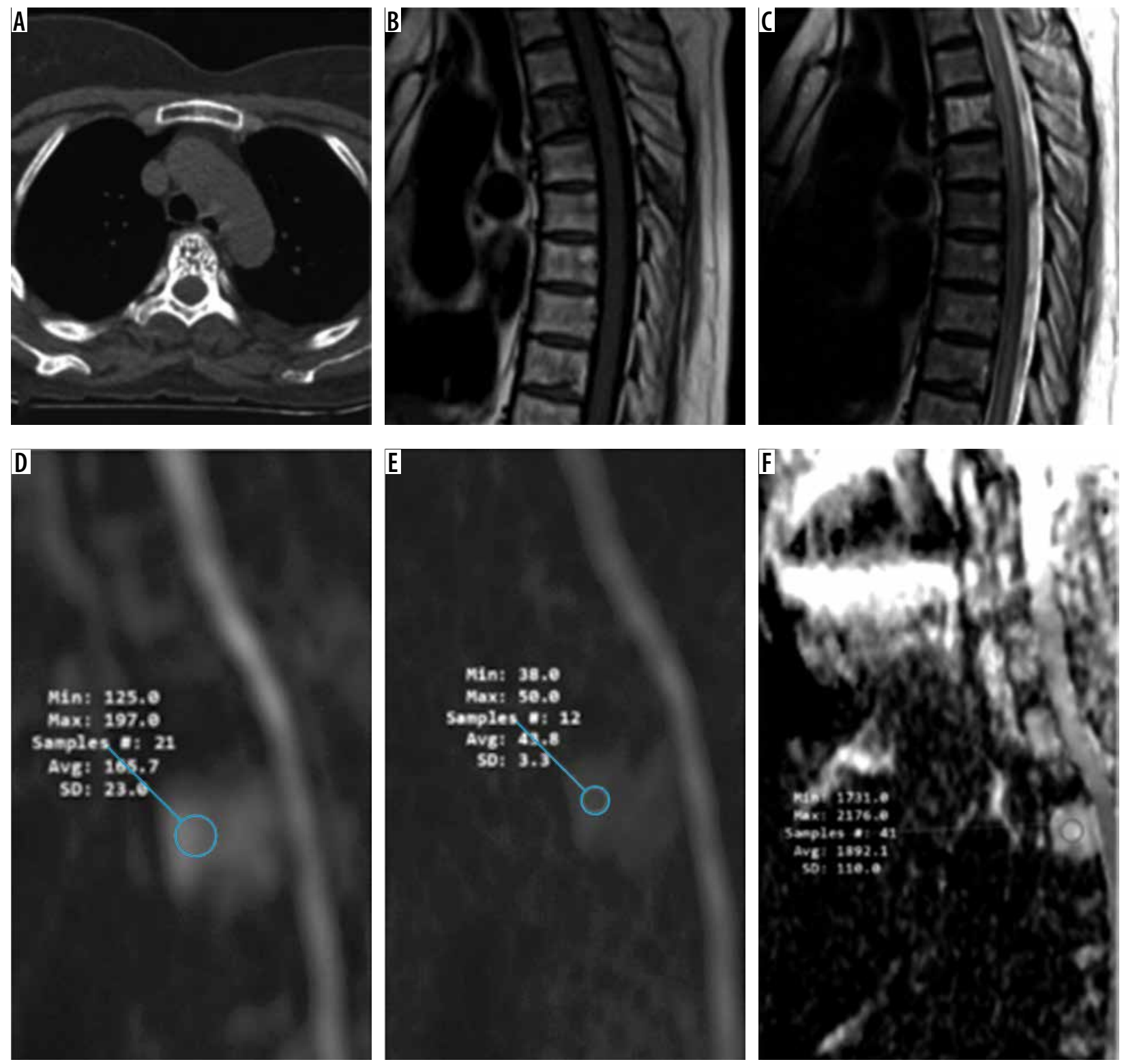

Figure 1. Atypical haemangioma in a 69-year-old woman. A) The characteristic polka-dot sign. B-C) The T1 and T2 sequences, respectively. The haemangioma is hypointense in T1- and hyperintense in T2-weighted images. D-F) Diffusion-weighted imaging in b values of 50, 400 , and the apparent diffusion coefficient (ADC) map. The ADC of the lesion equals $1892 \times 10^{-6} \mathrm{~mm}^{2} / \mathrm{s}$ 

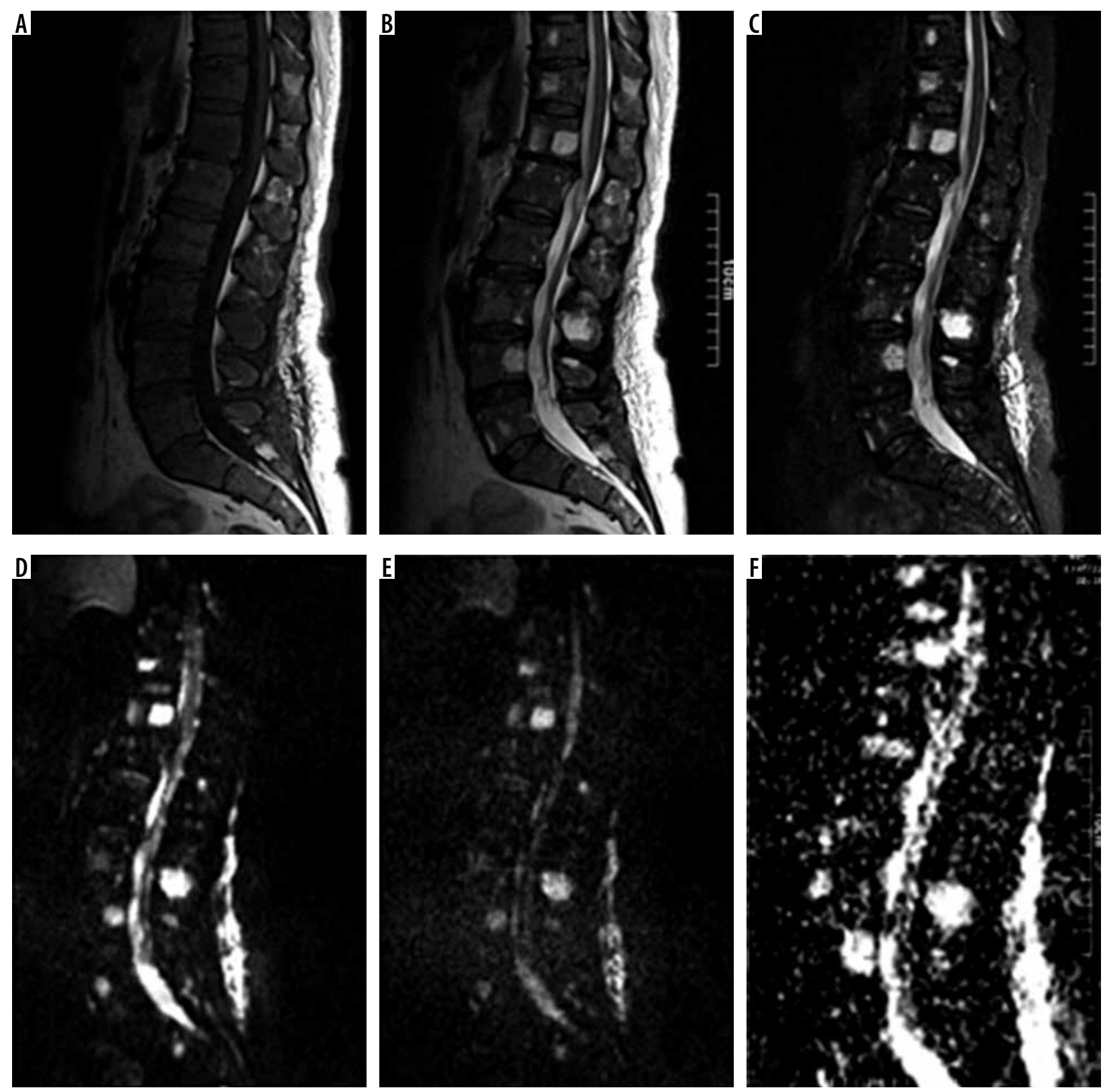

Figure 2. Focal multiple myeloma in a 47-year-old patient. A-C) Depict T1, T2, and TRIM sequences. D-F) Diffusion-weighted imaging in b values of 50, 400, and the apparent diffusion coefficient (ADC) map. The ADC value of the lesion equalled $1850 \times 10^{-6} \mathrm{~mm}^{2} / \mathrm{s}$

is a schematic presentation of imaging findings, and Figure 7. is a presentation of how the ROI was applied in ADC maps.

\section{Discussion}

In the present study it was shown that atypical haemangiomas had a significantly higher mean ADC value compared to malignant metastatic lesions. The mean \pm standard deviation of ADC values for atypical haemangiomas was 1888 $\pm 74\left(\times 10^{-6} \mathrm{~mm}^{2} / \mathrm{s}\right)$, while it was $1008 \pm 81\left(\times 10^{-6} \mathrm{~mm}^{2} / \mathrm{s}\right)$ for metastatic lesions. Importantly, there was little overlap between $\mathrm{ADC}$ values, and the minimum $\mathrm{ADC}$ value of atypical haemangiomas was higher than that of almost all malignant lesions. ROC curve analysis determined 958 $\times 10^{-6} \mathrm{~mm}^{2} / \mathrm{s}$ as the cut-off value to differentiate malignant lesions from benign ones.
MRI has long been used in imaging of the central nervous system, especially the spine and its adjacent structures. Haemangiomas are common findings in autopsies (up to $10 \%$ of cases) and are easily detected using CT scans and MRIs. Atypical haemangiomas, however, are harder to diagnose because they resemble malignant lesions on conventional T1 and T2 sequences. Based on previous experience on differentiating benign from malignant lesions of the spine using DW-MRI, scholars have suggested that the same principles could be used to differentiate metastatic lesions from atypical haemangiomas. A systemic review performed by Luo et al. investigated whether vertebral fractures occurring due to malignant lesions had significantly different ADC values compared to those resulting because of benign lesions. They included 12 studies with a total of 350 malignant lesions and 312 benign lesions 

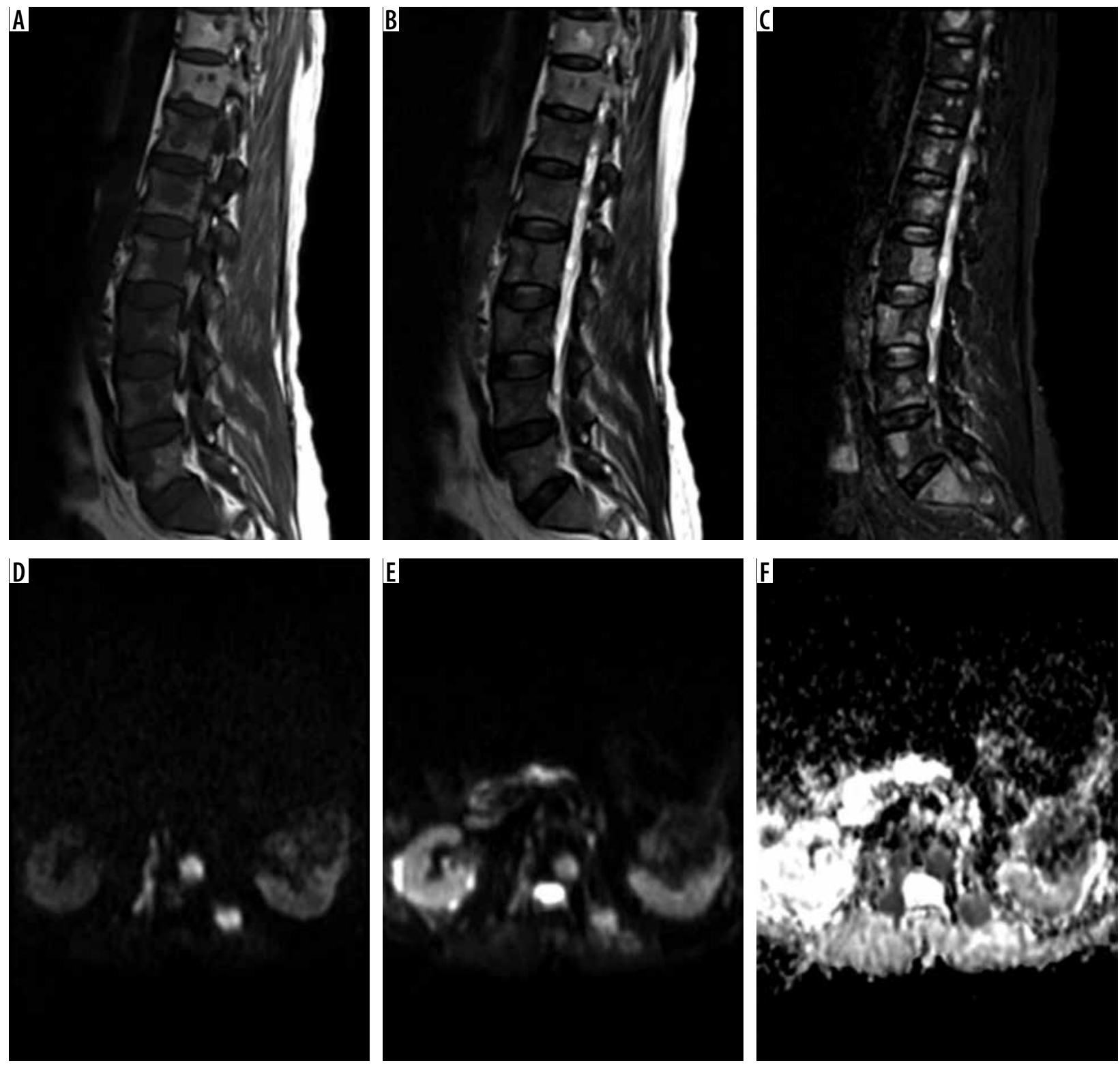

Figure 3. A metastasis, originating from renal cell carcinoma in a 46-year-old male. A-C) Show T1, T2, and TRIM sequences, respectively. D-E) Axial diffusion-weighted imaging cuts in b values of 50 and 400 . F) The apparent diffusion coefficient (ADC) map of the lesion. The ADC value of the lesion equalled $900 \times 10^{-6} \mathrm{~mm}^{2} / \mathrm{s}$

and found that the difference between the mean ADC value between the two groups was statistically significant. They also suggested that low b values (less than $500 \mathrm{~s} \mathrm{~mm}^{-2}$ ) were able to better differentiate these lesions from each other [11]. This article and similar ones were the reason for our choice of $b$ values.

Koutoulidis et al. investigated whether DW imaging could be used in the detection of multiple myeloma, and whether it could be used to differentiate focal and diffuse multiple myeloma. They found that the mean ADC values of normal vertebra, and vertebrae effected by focal and diffuse multiple myeloma, were significantly different $(0.360$ $\times 10^{-3} \mathrm{~mm}^{2} / \mathrm{s} \pm 0.110,1.046 \times 10^{-3} \mathrm{~mm}^{2} / \mathrm{s} \pm 0.232$, and 0.770 $\times 10^{-3} \mathrm{~mm}^{2} / \mathrm{s} \pm 0.135$, respectively) [12]. The findings of this study are in agreement with our observations, excluding the fact that the observed focal multiple myeloma in our study had a high ADC value compared to other malignant lesions, and even compared to the lesions studied by Koutoulidis et al.

Of note, usage of MRI may be indicated even more than previously expected in the diagnosis of atypical haemangiomas and their differentiation from multiple myelomas and metastasis, because studies have shown vertebral haemangiomas with positive PET scan results, making them a diagnostic challenge $[13,14]$. Interestingly, it seems that adverse clinical characteristics of haemangiomas may be related with their resemblance to malignant lesions in biomedical imaging, especially PET scans [15].

An early observation in this regard was made by Taşkin et al. They used DW-MRI to determine the ADC values of lesions found in 99 subjects. A total of 133 lesions were included, of which nine where atypical haemangiomas with 


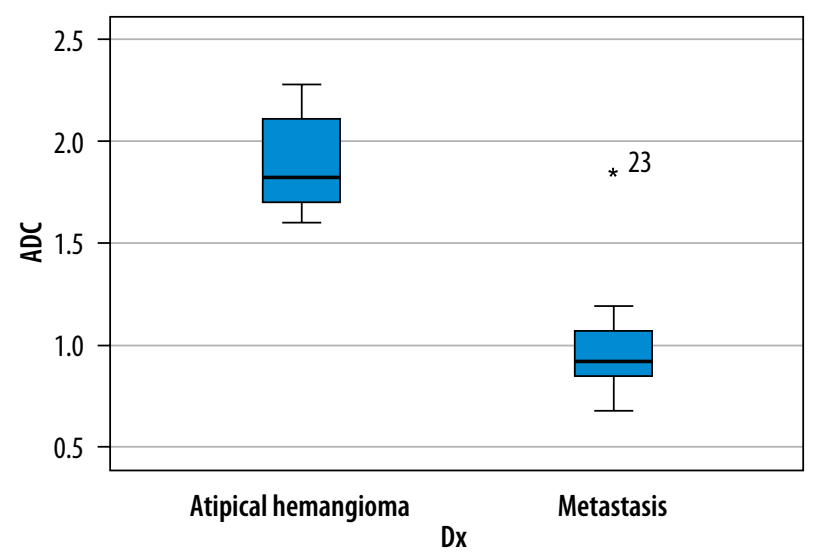

Figure 4. Plots and Wiggers diagram of the mean apparent diffusion coefficient $(A D C)$ values of atypical haemangiomas and malignant lesions

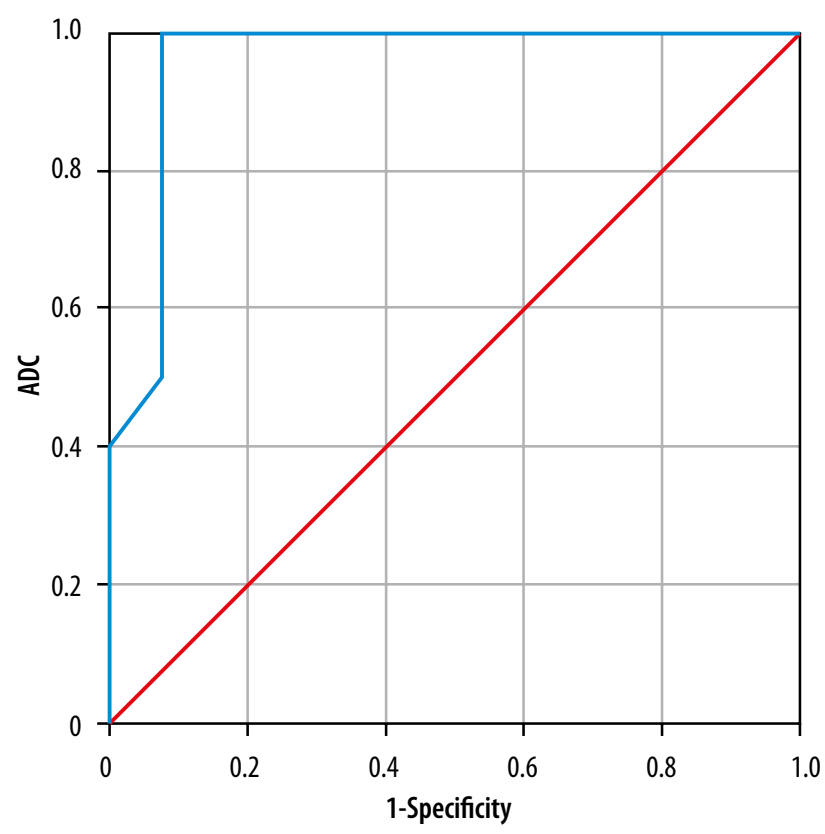

Figure 5. ROC curve analysis of the study

$\mathrm{T1}$
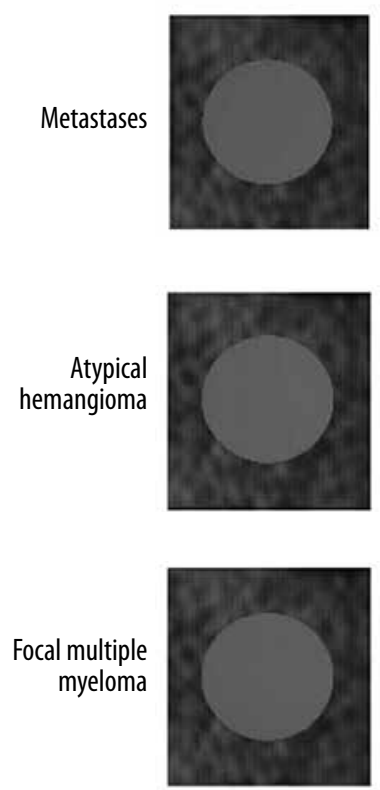

$\mathrm{T} 2$
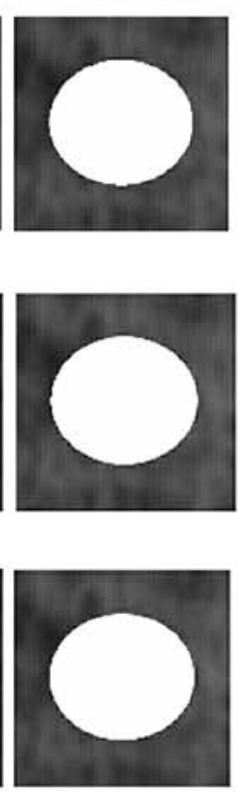

TIRM
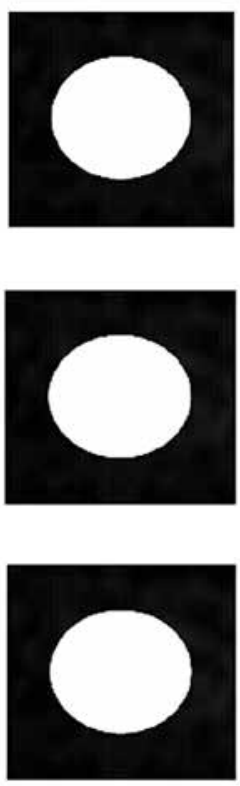

b value 50
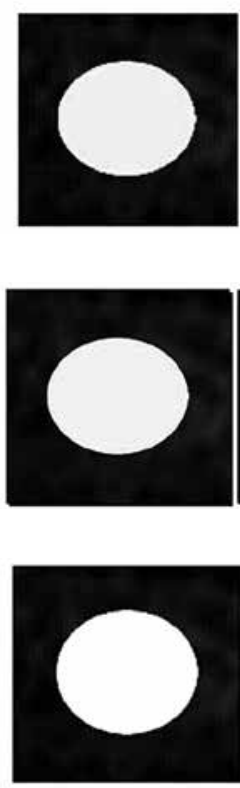

b value 400
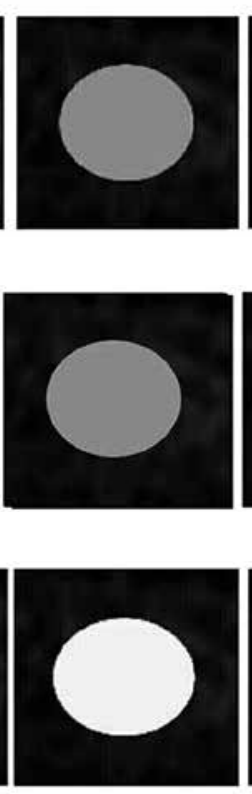

$A D C$
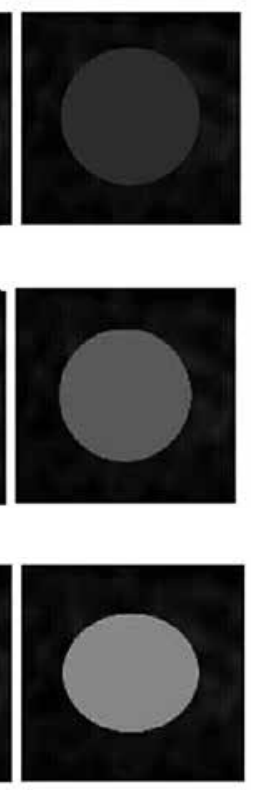

Figure 6. A summary of findings in different sequences of magnetic resonance imaging

a mean $\mathrm{ADC}$ value of $1.80 \pm 0.37\left(\times 10^{-3} \mathrm{~mm}^{2} / \mathrm{s}\right), 57$ were metastasis with a mean $\mathrm{ADC}$ value of $0.77 \pm 0.30\left(\times 10^{-3} \mathrm{~mm}^{2} / \mathrm{s}\right)$, and 17 were malignant compression fractures with a mean $\mathrm{ADC}$ value of $0.94 \pm 0.34\left(\times 10^{-3} \mathrm{~mm}^{2} / \mathrm{s}\right)$. The difference between the ADC values of malignant and benign lesions was statistically significant [16]. Similar results were also found by Shi et al., who compared the ADC values of atypical haemangiomas, typical haemangiomas, and metastasis [17].

Matrawy et al. studied the efficacy of DW-MRI in differentiating atypical haemangiomas from metastases, and also included typical haemangiomas in the study, as a separate group. A total of 24 patients were included: eight with metastasis, six with atypical haemangiomas, and 10 with typical haemangiomas. The mean ADC value for haemangiomas (including both kinds) was $1.54 \times 10^{-3} \mathrm{~mm}^{2} / \mathrm{s}$, and the mean ADC value of metastatic lesions was $0.83 \times 10^{-3} \mathrm{~mm}^{2} / \mathrm{s}$, with the difference being significant statistically. A threshold of $0.96 \times 10^{-3} \mathrm{~mm}^{2} / \mathrm{s}$ was suggested as being able to differentiate haemangiomas from malignant lesions [18]. The results of this study were in concordance with ours - we also found an identical cut-off point for differentiating different lesions. They found that atypical haemangiomas had a significantly higher mean ADC values compared to metastasis and had relatively high values compared to typical haemangiomas, although the mean was not significantly different between the two groups. Of note, a considerable overlap existed between the ADC values recorded for metastasis and atypical haemangiomas - a finding which is in contrast to ours. 

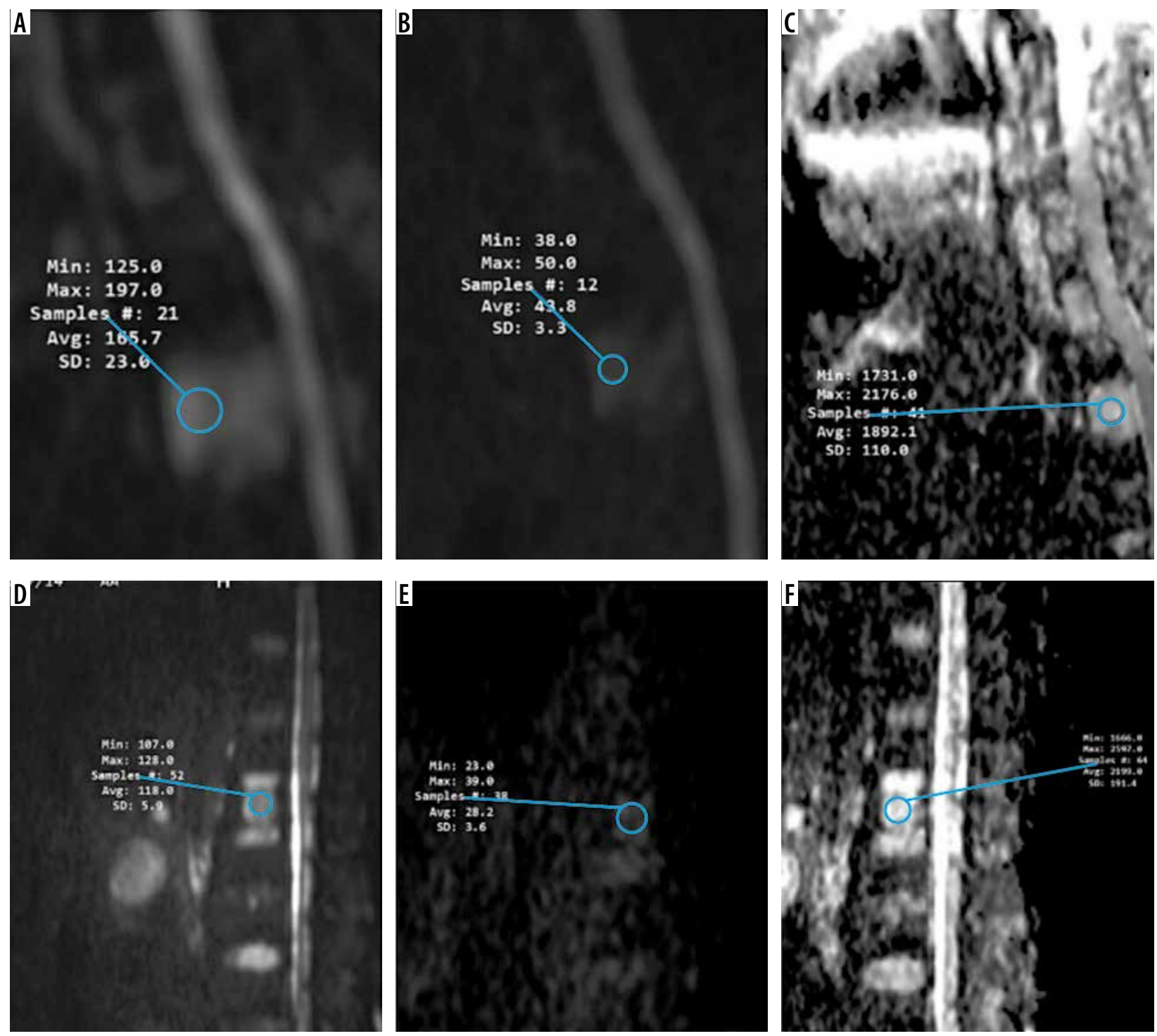

Figure 7. Apparent diffusion coefficient $(A D C)$ map of various lesions showing how $A D C$ values were defined for any given lesion. Measurements were made manually and for each lesion. Three separate measurements were made for each lesion, and the region of interest covered at least $50-75 \%$ of each lesion. Most measurements were made with an area of $0.5 \mathrm{~cm}^{2}$

In all of the previously mentioned studies, the number of patients with atypical haemangiomas ranged between 20 and 50 - a relatively small number with which to draw generalisable conclusions. We also faced the same limitation. Also, a limited number of metastatic lesions from limited types of tumours were involved, so our results may not be generalisable for making judgments regarding differentiation of atypical haemangiomas from certain types of malignancy or metastasis. This limitation was also seen in previous studies. More comprehensive multi-centre studies are needed to understand the exact benefit of DW imaging in differentiating atypical haemangiomas.

\section{Conclusions}

In the present cross-sectional study, we aimed to compare the mean ADC values for atypical haemangiomas and metastatic lesion in the vertebra. These lesions are nearly identical in T1 and T2 sequences and, if applicable, DW imaging may be of great benefit in differentiating these lesions. The mean $\mathrm{ADC}$ value for atypical haemangiomas was $1888 \pm 74\left(\times 10^{-6} \mathrm{~mm}^{2} / \mathrm{s}\right)$, and the mean for metastatic lesions was $1008 \pm 81\left(\times 10^{-6} \mathrm{~mm}^{2} / \mathrm{s}\right)$, with the difference being statistically significant. ROC curve analysis revealed that an ADC value of $958 \times 10^{-6} \mathrm{~mm}^{2} / \mathrm{s}$ could differentiate benign lesions from metastatic ones. Considering the limitations of this study, the findings show that DW imaging could be useful in differentiating atypical haemangiomas from metastatic lesions.

\section{Conflict of interest}

The authors report no conflict of interest. 


\section{References}

1. Slon V, Stein D, Cohen H, et al. Vertebral hemangiomas: their demographical characteristics, location along the spine and position within the vertebral body. European Spine J 2015; 24: 2189-2195.

2. Persaud T. The polka-dot sign. Radiology 2008; 246: 980-981.

3. Ross JS, Masaryk TJ, Modic MT, et al. Vertebral hemangiomas: MR imaging. Radiology 1987; 165: 165-169.

4. Rodallec MH, Feydy A, Larousserie F, et al. Diagnostic imaging of solitary tumors of the spine: what to do and say. Radiographics 2008 28: 1019-1041

5. Suh CH, Choi YJ, Baek JH, et al. The diagnostic value of diffusionweighted imaging in differentiating metastatic lymph nodes of head and neck squamous cell carcinoma: a systematic review and meta-analysis. AJNR Am J Neuroradiol 2018; 39: 1889-1895.

6. Morales KA, Arevalo-Perez J, Peck KK, et al. Differentiating atypical hemangiomas and metastatic vertebral lesions: the role of T1-weighted dynamic contrast-enhanced MRI. AJNR Am J Neuroradiol 2018; 39: 968-973.

7. Bellasri S, Fatihi J, Elktaibi A, et al. Acute spinal cord compression caused by atypical vertebral hemangioma. J Craniovertebr Junction Spine 2017; 8: 275-277.

8. Bammer R. Basic principles of diffusion-weighted imaging. Eur J Radiol 2003; 45: 169-184.

9. White NS, McDonald CR, Farid N, et al. Diffusion-weighted imaging in cancer: physical foundations and applications of restriction spectrum imaging. Cancer Res 2014; 74: 4638-4652.

10. Nerad E, Pizzi AD, Lambregts DMJ, et al. The Apparent Diffusion Coefficient (ADC) is a useful biomarker in predicting metastatic colon cancer using the ADC-value of the primary tumor. PloS One 2019; 14: e0211830
11. Luo Z, Litao L, Gu S, et al. Standard-b-value vs low-b-value DWI for differentiation of benign and malignant vertebral fractures: a metaanalysis. Br J Radiol 2016; 89: 20150384.

12. Koutoulidis V, Fontara S, Terpos E, et al. Quantitative Diffusionweighted Imaging of the Bone Marrow: An Adjunct Tool for the Diagnosis of a Diffuse MR Imaging Pattern in Patients with Multiple Myeloma. Radiology 2017; 282: 484-93.

13. Solav SV, Savale SV, Patil AM. False-positive FDG PET CT Scan in Vertebral Hemangioma. Asia Ocean J Nucl Med Biol 2019; 7: 95-98.

14. Usmani S, Marafi F, Rasheed R, et al. Atypical hemangioma mimicking metastasis on 18F-sodium fluoride positron emission tomography-computed tomography and magnetic resonance imaging: gallium-68-prostate-specific membrane antigen positron emission tomography improves the specificity of bone lesions. Indian J Nucl Med 2018; 33: 171.

15. Bagul M, Mehra R. Pre and post radiotherapy imaging findings of aggressive vertebral haemangioma of the thoracic spine. J Case Rep 2016; 6: 280-283.

16. Taşkin G, Incesu L, Aslan K. The value of apparent diffusion coefficient measurements in the differential diagnosis of vertebral bone marrow lesions. Turk J Med Sci 2013; 43: 379-387.

17. Shi YJ, Li XT, Zhang XY, et al. Differential diagnosis of hemangiomas from spinal osteolytic metastases using 3.0 T MRI: comparison of T1-weighted imaging, chemical-shift imaging, diffusion-weighted and contrast-enhanced imaging. Oncotarget 2017; 8: 71095-71104.

18. Matrawy KA, El-Nekeidy AA, El-Sheridy HG. Atypical hemangioma and malignant lesions of spine: can diffusion weighted magnetic resonance imaging help to differentiate? The Egypt J Radiol Nucl Med 2013; 44: 259-263. 